\title{
Determining the spacetime structure of bottom-quark couplings to spin-zero particles
}

\author{
Tathagata Ghosh, ${ }^{1, *}$ Rohini Godbole, ${ }^{2, \dagger}$ and Xerxes Tata ${ }^{1, \$}$ \\ ${ }^{1}$ Department of Physics and Astronomy, University of Hawaii, Honolulu, Hawaii 96822, USA \\ ${ }^{2}$ Centre for High Energy Physics, Indian Institute of Science, Bangalore, 560012, India
}

(Received 27 April 2019; published 18 July 2019)

\begin{abstract}
We present a general argument that highlights the difficulty of determining the spacetime structure of the renormalizable bottom-quark Yukawa interactions of the Standard Model Higgs boson, or for that matter of any hypothetical spin-zero particle, at high energy colliders. The essence of the argument is that, it is always possible, by chiral rotations, to transform between scalar and pseudoscalar Yukawa interactions without affecting the interactions of bottom quarks with SM gauge bosons. Since these rotations affect only the $b$-quark mass terms in the Standard Model Lagrangian, any differences in observables for scalar versus pseudoscalar couplings vanish when $m_{b} \rightarrow 0$, and are strongly suppressed in high energy processes involving the heavy spin-zero particle where the $b$ quarks are typically relativistic. We show, however, that the energy dependence of, for instance, $e^{+} e^{-} \rightarrow b \bar{b} X$ (here $X$ denotes the spin-zero particle) close to the reaction threshold may serve to provide a distinction between the scalar versus pseudoscalar coupling at electron-positron colliders that are being proposed, provided that the $X b \bar{b}$ coupling is sizeable. We also note that while various kinematic distributions for $t \bar{t} h$ are indeed sensitive to the spacetime structure of the top-Yukawa coupling, for a spin-zero particle $X$ of an arbitrary mass, the said sensitivity is lost if $m_{X} \gg m_{t}$.
\end{abstract}

DOI: 10.1103/PhysRevD.100.015026

\section{INTRODUCTION}

With the discovery of the spin-zero Higgs-like boson $h$ in 2012 [1] at the CERN LHC, the entire particle content of the Standard Model (SM) has been experimentally observed. That this new particle plays a role in electroweak symmetry breaking is clear from the existence of $h Z Z$ and $h W^{+} W^{-}$couplings whose spacetime structure and size are close to SM expectations [2], with only (loop-level) suppressed deviations from their SM values possible. All measurements of the properties of $h$, to date, are compatible with $h$ being the SM Higgs boson. These include measurements of couplings of $h$ to third generation fermions, tau [3], bottom [4] and top [5], and an upper limit on the muon Yukawa coupling just a factor of 2-3 above its SM expectation [6].

We stress that while the properties of $h$ are perfectly compatible with those of the SM Higgs boson, the spacetime structure of its couplings to fermions is as yet

\footnotetext{
*tghosh@hawaii.edu

†rohini@iisc.ac.in

†tata@phys.hawaii.edu
}

Published by the American Physical Society under the terms of the Creative Commons Attribution 4.0 International license. Further distribution of this work must maintain attribution to the author(s) and the published article's title, journal citation, and DOI. Funded by SCOAP . very poorly determined. The possibility that $C P$ violation (CPV) may be present in $h f \bar{f}$ couplings at tree level, via the interaction terms,

$\mathcal{L}_{\mathrm{int}}=\bar{f}\left(c_{f}+i d_{f} \gamma_{5}\right) f h=y_{f} \bar{f}\left(\cos \alpha_{f}+i \sin \alpha_{f} \gamma_{5}\right) f h$,

is not experimentally excluded. Indeed CPV interactions of spin-zero particles with fermions could arise in models where $h$ comprises both $C P$ even and $C P$ odd components. In contrast, $C P$-violating $h V V(V=W, Z)$ interactions can arise only at the loop level as long as $C P$ is not violated spontaneously and will reflect themselves as nonrenormalizable, higher-dimensional operators.

LHC constraints from the measurements of various Higgs boson decay rates on CPV top-quark Yukawa interactions have been examined in Ref. [7]. These authors and many others [8-16] analyze various kinematic distributions, spin correlations and $C P$-violating observables that would be possible to measure at the LHC and show that these could be used to constrain $\alpha_{t}$ in Eq. (1). Indeed, the spacetime structure of the top-Yukawa interaction may also be probed via studies of top polarization and $\mathrm{CPV}$ asymmetries of top decay products in $e^{+} e^{-} \rightarrow t \bar{t} h$ at electron-positron colliders [8,9]. Many groups [10] have suggested $h \rightarrow \tau^{+} \tau^{-}$decays and constructed CPV asymmetries out of $\tau^{ \pm}$polarization vectors (some directly 
observable, others proxies for observable quantities) that may be used to restrict the value of $\alpha_{\tau}$ at the LHC.

While considerable attention has been devoted to the structure of the top and tau Yukawa couplings, the bottom Yukawa has received only limited attention [11,12]. Gunion and $\mathrm{He}$ [11] note that an explicit evaluation of the $f \bar{f} h$ production from the $g g$ or $q \bar{q}$ initial state shows that the $\alpha_{f}$-dependent terms are proportional to $m_{f}^{2}$ (not counting any $m_{f}$ factors in the Yukawa coupling), and so significant only for $f=t$ (since for other quarks the $m_{f}^{2}$ term is negligible compared with typical dot products of four-momenta in the process) which they then focus on. Gritsan et al. [12] examine several parton-level kinematic distributions for the $b b h$ final state and confirm that there is essentially no detectable dependence on $\alpha_{b}$.

Initially, our goal was to examine the prospects for determining the spacetime structure of the (renormalizable) bottom-quark Yukawa couplings of the Higgs-like boson via measurements at high energy colliders such as the LHC or a future electron-positron Higgs boson factory. During the course of our study, we arrived at an understanding (based on chirality arguments presented in Sec. II) as to why earlier studies $[11,12]$ found no observable dependence on $\alpha_{b}$. We find that while the small Yukawa coupling certainly makes things more difficult, the real underlying reason is the smallness of the $b$ mass relative to the energy scale of the hard scattering process (set by $m_{h}$ ). Put differently, our arguments clearly illustrate the issues with determining the spacetime structure of the Yukawa interaction of a spin-zero hypothetical particle $X$ to relatively light fermions, even if the associated coupling is order unity. While our conclusions about prospects for determining the spacetime structure of the Yukawa coupling of the bottom quark are largely negative (see Sec. IV for an exception to the general arguments), and only confirm the findings via explicit computations in the literature, we felt that the deeper understanding that we have gained of the underlying reason for this is worthwhile to report.

The chirality arguments that lead us to conclude that no physics can depend on $\alpha_{f}$ are valid in the limit $m_{f} \rightarrow 0$, and break down when fermion mass effects are important, i.e., when the fermion is nonrelativistic, as is the case close to the kinematic threshold for the production process. With this in mind, we also examine the threshold behavior of $2 \rightarrow 3 X b \bar{b}$ processes with a large value of $X b \bar{b}$ coupling in Sec. IV. These results, which we believe to be new, offer an in-principle way of distinguishing $\alpha_{f}=0$ from $\alpha_{f}=\pi / 2$ at, e.g., an $e^{+} e^{-}$collider operating just above the energy threshold for $b \bar{b} X$ production.

The remainder of this paper is organized as follows. In Sec. II we present our argument based on chirality to demonstrate that the angle $\alpha_{f}$ becomes unobservable in the limit that the fermion mass vanishes. This then provides a dynamical understanding of the results in Refs. [11,12] where explicit computations showed that the effects of $\alpha_{b}$ in $h b \bar{b}$ production at the LHC are too small to be observed, even though corresponding studies of $t \bar{t} h$ production illustrate techniques for the determination of $\alpha_{t}$. Our argument also illustrates why it will not be possible to extract $\alpha_{b}$ using kinematic variables in any process at high energy facilities. In the next section, we provide explicit illustrations of the chirality arguments of Sec. II, for both the SM Higgs boson and the hypothetical spin-zero $X$ boson with large Yukawa couplings mentioned above. In Sec. IV we derive the threshold behavior of $2 \rightarrow 3$ processes and apply our results to $b \bar{b} X$ production processes, where $X$ couples to the $b \bar{b}$ pair as in Eq. (1), either with $\alpha_{f}=0$ or with $\alpha_{f}=\pi / 2$. In Sec. V we digress from the main theme of this paper. We note that while the various kinematic distributions in $t \bar{t} h$ are indeed sensitive to the spacetime structure of the top-Yukawa coupling, the same is not true for a spin-zero particle $X$ of an arbitrary mass. The said sensitivity is lost for $m_{X} \gg m_{t}$. We end in Sec. VI with a summary of our results and some general conclusions.

\section{CHIRALITY CONSIDERATIONS AND CP VIOLATION}

We begin by noting that the Lagrangian in Eq. (1) can be rewritten after chiral transformations,

$$
b_{R, L}=e^{i \theta_{R, L}^{b}} b_{L, R}^{\prime}
$$

as

$$
\mathcal{L}_{\text {int }}=\overline{b_{R}^{\prime}} e^{-i\left(\theta_{R}^{b}-\theta_{L}^{b}\right)} e^{-i \alpha_{b}} b_{L}^{\prime} h+\text { H.c. }
$$

We thus see that by choosing $\theta_{L}^{b}-\theta_{R}^{b}=\alpha_{b}$, we can rotate away the $C P$-violating term in the Lagrangian in Eq. (1). Of course, such a chiral transformation would lead to a pseudoscalar bilinear term proportional to $m_{b}$, and we would have achieved nothing. However, if $m_{b}=0$, there would be no such term. It would then appear that any $C P$ violating effects from the Lagrangian in Eq. (1) must vanish if $m_{b}=0$.

Note that the charged and neutral current couplings to the vector bosons of the SM are also unaltered, as long as we perform a common chiral transformation for the electroweak doublet, i.e., we take $\theta_{L}^{t}=\theta_{L}^{b} \equiv \theta_{L}$ [as we must to preserve $S U(2)_{L}$ ]. We still have the freedom to make arbitrary chiral transformations on $t_{R}$, since there is no right-handed charged vector current in the SM. We can then use this freedom and choose $\theta_{R}^{t}=\theta_{L}$ to keep the topquark mass term in the standard form. Any contribution of the form of (1) to the top-quark sector would be left unaltered. If the top-quark Yukawa has the SM form (i.e., $\alpha_{t}=0$ ), any effect of the spacetime structure of the bottom-quark Yukawa coupling must vanish as $m_{b} \rightarrow 0$. In particular, any $\alpha_{b}$ dependence in $t b h$ production processes vanish with the $b$ mass. 
We thus conclude that in the $\mathrm{SM}$, any $C P$-violating effects from the Lagrangian in Eq. (1) with $f=b$ must vanish if $m_{b}=0$. This observation, which may well be known to some aficionados, provides a clear explanation of the negative results $[11,12]$ about the prospects to observe $\sin \alpha_{b}$ effects in bottom Higgs Yukawa couplings, even though corresponding effects are readily observable in the top-Yukawa sector [7,11-19]. Indeed, because our argument is made at the Lagrangian level, it applies not only to these processes, but to essentially any process that may be envisioned to study $C P$-violating effects from bottomquark Higgs boson Yukawa interactions. Moreover, since we did not use any properties of $h$ (other than its spin) in arriving at our result, our argument also applies to the corresponding couplings of any spin-zero neutral particle $X$ to SM fermions, at least for processes that do not simultaneously involve also the SM Higgs boson Yukawa coupling. We will refer to this as chirality protection of $C P$ invariance in the Yukawa sector.

\section{ILLUSTRATION OF CHIRALITY PROTECTION}

\section{A. The Standard Model Higgs particle}

Prospects for the exploration of the spacetime structure of SM top interactions, both at the LHC and at electronpositron colliders, have been examined by many authors [7-9,11-22]. Many potential observables have been suggested for the determination of $\alpha_{t}$. These observables naturally divide up into kinematic quantities such as transverse momenta, angles, or invariant masses, and polarization observables which depend on the fact that the top-quark decays very rapidly (compared to the hadronization time) so that polarization information is maintained by its decay products. In contrast, the bottom quark typically hadronizes to excited $b$ hadrons, which deexcite to lighter $b$ hadrons whose decay rates to yet lighter states are (dynamically and/ or kinematically) suppressed and so may compete with the $b$-quark spin flip rate. As a result, information of the $b$ polarization in the hard production process is largely screened from the final bottom meson decay products [23]. ${ }^{1}$ Since $b$-quark Yukawa interactions are the main subject of this paper, we focus our attention on kinematic observables from this point on.

Clearly, along with $h \rightarrow \tau^{+} \tau^{-}$decay, $f \bar{f} h$ production offers the best prospects for studying the spacetime structure of the Yukawa interactions of $h$ using kinematic distributions. Before turning to the discussion of the bottom Yukawa coupling, we quickly review what has been done for the much-more-accessible, and therefore, more studied top-Yukawa interaction. There are numerous studies of $t \bar{t} h$

\footnotetext{
${ }^{1}$ It has been noted $[23,24]$ that $\Lambda_{b}$ baryons partially preserve the original $b$-quark polarization. However, since the probability of $b$-quark fragmentation to baryons is just a few percent, we do not consider this further in our study.
}

process at the LHC. Kinematic variables, many at parton level, have been constructed using the momenta of the $t, \bar{t}$ and $h$ and hence can be constructed in the laboratory frame. Various angular observables from the momenta of the $t$ decay products have also been constructed, both in the laboratory frame as well as some special frames such as the $t \bar{t}$ rest frame, as they carry information about spin-spin correlation between the $t$ and $\bar{t}$ which in turn is affected by the value of $\alpha_{t}$. All these observables while being $C P$ even, have the potential to distinguish between $\alpha_{t}=0$ and $\alpha_{t}=\pi / 2$. These include

(i) $M_{t \bar{t} h}, p_{T_{h}}, \Delta \phi(t, \bar{t})[7,12,13,18]$

(ii) $M_{t \bar{t}}, M_{t h}[13]$

(iii) $p_{T_{h}}, p_{T_{t}}, M_{t \bar{t}}[14]$

(iv) Pseudorapidity differences between the (anti)leptons or bottom quarks coming from the $t$ and $\bar{t}$ decay respectively, for an $h$ with high transverse momentum: $\Delta \eta_{l^{+} l^{-}}, \Delta \eta_{b \bar{b}}[15]$.

(v) The lab-frame angle $\Delta \theta^{l h}\left(l^{+} l^{-}\right)$between $l^{+}$and $l^{-}$ projected onto a plane perpendicular to the $h$ direction [7].

(vi) $\Delta \phi^{t, \bar{t}}\left(l^{+}, l^{-}\right)$: The difference between the azimuthal angles of the $l^{+}$in the rest frame of $t$ and that of $l^{-}$in the rest frame of $\bar{t}[7,13,15,18,19]$.

(vii) $M_{t \overline{t h}}, \theta_{t}, \Phi_{t}^{*}, M_{t \bar{t}}, \theta_{h}, \theta_{b}, \Phi_{b}$ [12]. Here, $\theta_{t}$ is the angle between the top-quark direction and the opposite of the Higgs direction in the $t \bar{t}$ frame; $\Phi_{t}^{*}$ is the angle between the decay planes of the $t \bar{t}$ system and $h \rightarrow f \bar{f}$ in the $t \bar{t} h$ frame; $\theta_{b}$ is the angle between the $b$ quark and opposite of the $W^{+} W^{-}$ system in the $b \bar{b}$ frame, and finally, $\Phi_{b}$ is the angle between the planes of the $b \bar{b}$ and $W^{+} W^{-}$systems in the $t \bar{t}$ frame. This paper also examines many other distributions, but we have picked out the ones that appear to give maximum distinction between $\alpha_{t}=0$ and $\alpha_{t}=\pi / 2$.

In addition, several $C P$-violating asymmetries $[7,13,16,17]$ have been proposed for the purpose of extracting $\alpha_{t}$. Since these are not directly relevant to us, we do not list these here. Very recently, Goncalves et al. [25] have examined the structure of the top-Yukawa coupling using variables related to $M_{T 2}$ to aid in the selection of signal events.

To illustrate the degree of distinction between $\alpha_{t}=0$ and $\alpha_{t}=\pi / 2$ that may be possible at the LHC, we show in Fig. 1 the idealized $p_{T h}$ distribution for $p p \rightarrow t \bar{t} h+X$ events, taking $m_{h}=125 \mathrm{GeV}$. In the left panel, we show the differential distribution assuming that the Yukawa coupling is given by its SM value for both the scalar $\left(\alpha_{t}=0\right.$, solid blue line) and for the pseudoscalar $\left(\alpha_{t}=\pi / 2\right.$, dashed red line) cases. The shapes, as well as the overall normalizations provide a clear distinction between the two cases. Since the case $\alpha_{t}=\pi / 2$ would clearly be for a new particle with an unknown coupling, it is not reasonable to use the absolute normalizations to distinguish between the two cases. With this in mind, we show the corresponding distributions 

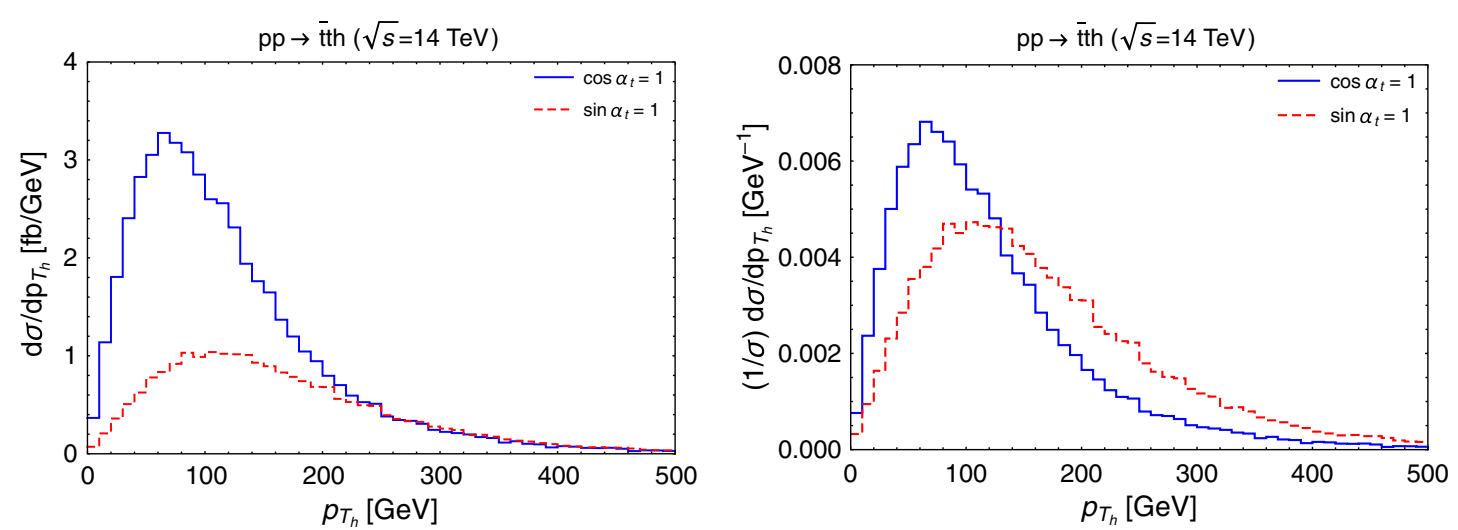

FIG. 1. Left panel: The distribution of $p_{T_{h}}$ in $p p \rightarrow t \bar{t} h$ production at the LHC with $\sqrt{s}=14 \mathrm{TeV}$, assuming that the Lagrangian Yukawa coupling is given by its SM value for both $\alpha_{t}=0$ as well as $\alpha_{t}=\pi / 2$. Right panel: The same distribution normalized to unity. The total cross sections for the two cases are 480.6 and $219.6 \mathrm{fb}$, respectively.

normalized to unity in the right-hand frames. We see that the shapes alone provide a clear distinction, and so to the extent that it is possible to reliably determine $p_{T h}$ in LHC events (this is not the subject of this paper), it should be possible to distinguish between the scalar and the pseudoscalar cases, and perhaps also obtain a measure of $\alpha_{t}$. We present these results here for completeness and have checked that they agree with the results available in the literature, e.g., in Ref. [7].

Prospects for examining the spacetime structure of the top-quark Yukawa coupling via $e^{+} e^{-} \rightarrow t \bar{t} h$ production have also been examined by numerous groups. The most promising ways include the dependence of the total cross section on the center-of-mass energy [9,21], a study of kinematic variables $E_{W}, E_{h}$ [21], polarization asymmetries [9] and toponium production in association with $h$ [22]. We refer the interested reader to the literature for details.

We begin our discussion of $p p \rightarrow b \bar{b} h$ at LHC14 by showing the distribution of the transverse momentum of the $h$ boson in these events in Fig. 2, again assuming that the bottom Yukawa coupling is given by its $\mathrm{SM}$ value. We require $E_{T}(b)>30 \mathrm{GeV}$, and $|\eta(b)|<2.5$ to very roughly capture $b$-jet identification effects. We do not attempt to impose $b$-jet tagging efficiencies, so the cross sections shown should be regarded as overestimates. There is one significant difference from the corresponding $p p \rightarrow t \bar{t} h$ production case shown in the previous figure that is worth mentioning. Because of the smallness of the bottom Yukawa coupling, electroweak production where the $h$ is radiated off the (virtual) $Z$ boson now makes a comparable contribution after $b$ quark $E_{T}$ and rapidity cuts for the case with $\alpha_{b}=0 .{ }^{2}$ Here, and in the remainder of this paper, we

\footnotetext{
${ }^{2}$ Without any $E_{T}(b)$ and $\eta(b)$ cuts the cross sections for the $\cos \alpha_{b}=1, y_{b}=0$ case is $90.4 \mathrm{fb}$ while the corresponding total cross section is $946 \mathrm{fb}$. This is to be compared with $37.1 \mathrm{fb}$ for the $\cos \alpha_{b}=1, y_{b}=0$ case and a total cross section of $51.4 \mathrm{fb}$ that one obtains after cuts on $E_{T}(b)$ and $\eta(b)$.
}

assume that the couplings of the spin-zero particle to vector bosons (which have been reasonably well determined) do not violate $C P$, so that there is no $h Z Z$ coupling when $\alpha_{b}=\pi / 2$. As in Fig 1, we show two frames, with the absolute cross sections on the left, and cross sections normalized to unity on the right. For the $\alpha_{b}=0$ case, we show three histograms to separate out the pure electroweak contribution where the $h$ is radiated off the $Z^{*}$, which is absent in the $\alpha_{b}=\pi / 2$ case.

We see that there is a significant difference between the full $\alpha_{b}=0$ (blue, solid) and $\alpha_{b}=\pi / 2$ (green, dot-dashed) histograms in both the left and right frames, suggesting that it is possible to distinguish between the two cases. We note, however, that the differences are very sensitive to the details of the cuts that we impose on the $b$ parton. Given our very rudimentary treatment of $b$ jets these will also not reflect the experimental $b$-jet distributions. More importantly, these differences arise almost entirely from the $Z Z h$ coupling, and do not reflect the difference between $\alpha_{b}=0$ and $\alpha_{b}=\pi / 2$. Indeed we see that the normalized $\alpha_{b}=\pi / 2$ (green, dot-dashed) histogram closely tracks the corresponding $\alpha_{b}=0$ with $Z Z h$ coupling switched off (red dashed) histogram in the right panel, confirming that the differences of shape arise mostly from the additional $Z Z h$ coupling that is present for $\alpha_{b}=0$. This is an explicit realization of our general result in Sec. II that any difference (other than due to the $h Z Z$ coupling) between $\alpha_{b}=0$ and $\alpha_{b}=\pi / 2$ should vanish as $m_{b} \rightarrow 0$. Although we have shown this for just the $p_{T h}$ distribution, we mention in passing that similar results are obtained for other kinematic variables.

Next, we turn to the examination of prospects for determining $\alpha_{b}$ at $e^{+} e^{-}$colliders along the lines of corresponding studies for $\alpha_{t}[9,21]$. Toward this end, we consider $e^{+} e^{-} \rightarrow b b h$ production at future electron-positron colliders, which have been proposed for precision studies of $Z$ and Higgs bosons, and of the $W W$ threshold [26]. This process occurs via amplitudes for $b \bar{b}$ production 

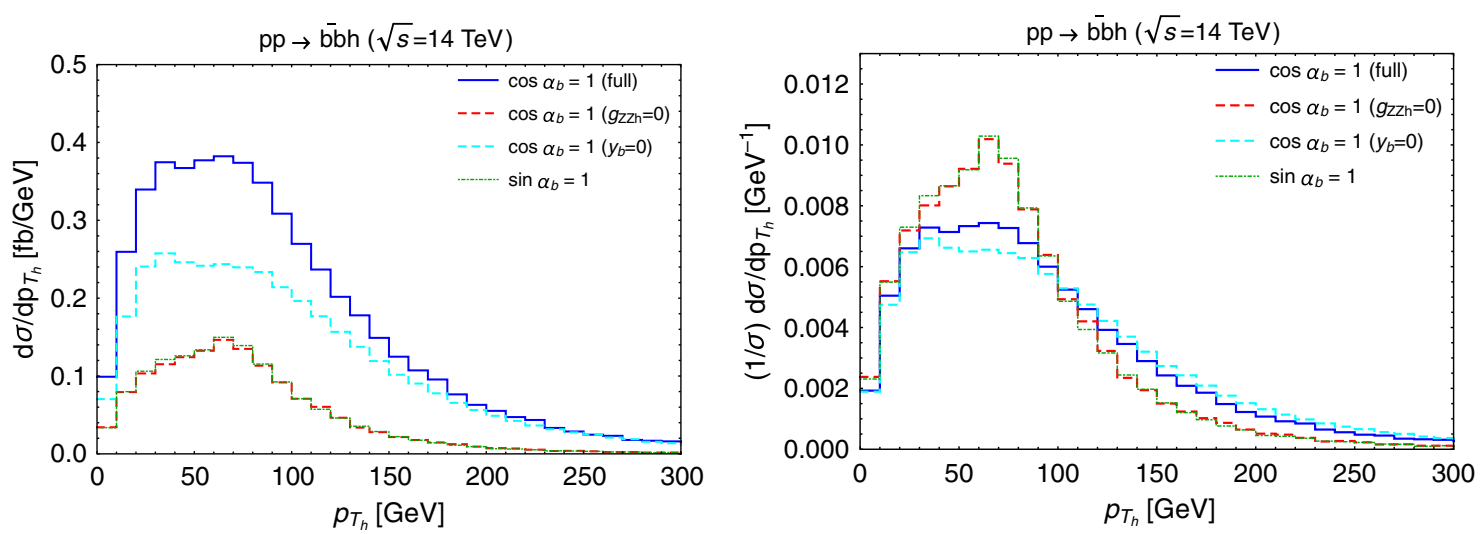

FIG. 2. Left panel: The distribution of $p_{T_{h}}$ in $p p \rightarrow b \bar{b} h$ production at the LHC with $\sqrt{s}=14$ TeV, assuming that the Lagrangian Yukawa coupling is given by its SM value for both $\alpha_{b}=0$ as well as $\alpha_{b}=\pi / 2$. Right panel: The same distribution normalized to unity. The purely electroweak contribution where the $h$ is radiated off the virtual $Z$ boson is significant in this case. After the $E_{T}(b)>30 \mathrm{GeV}$ and $|\eta(b)|<2.5$ cuts, the cross section for the case $\cos \alpha_{b}=1$ and $y_{b}=0$ is $37.1 \mathrm{fb}$ while the corresponding total cross section is $51.4 \mathrm{fb}$. The impact of the ZZh coupling, which we assume is absent for the $\alpha_{b}=\pi / 2$ case, is illustrated by the three histograms shown.

via a virtual $Z$, and where the $h$ is radiated off either the quark line, or (for $\alpha_{b} \neq \pi / 2$ ) off the $Z^{*}$. We focus on operation at a center-of-mass energy $\sqrt{s}=161 \mathrm{GeV}$, since the process is kinematically inaccessible for $\sqrt{s}=M_{Z}$, and dominated by $2 \rightarrow 2 e^{+} e^{-} \rightarrow h Z(\rightarrow b \bar{b})$ production at the higher energy $\sqrt{s}=250 \mathrm{GeV}$ envisioned for detailed Higgs boson study. The integrated luminosity per interaction region is envisioned to be $\sim 1.3(3.8) \mathrm{ab}^{-1} / \mathrm{yr}$ for the Circular Electron Positron Collider (CEPC) [Future Circular Collider (FCC)] design [26].

As for the LHC studies just discussed, we show the $p_{T h}$ distribution in Fig. 3, again with the left (right) panels for absolute values of the distributions (distributions normalized to unity). As in Fig. 2 we assume that the $h Z Z$ coupling vanishes if $\alpha_{b}=\pi / 2$, and show three histograms for $\alpha_{b}=0$ but just one histogram for $\alpha_{b}=\pi / 2$. From the left panels, we see instantly that the total cross section of $17.2 \mathrm{ab}$ for $\alpha_{b}=0$ is completely dominated by the $Z Z h$ coupling, with per mille size contributions $\simeq 9.75 \times$ $10^{-3} \mathrm{ab}$ (read off on the right-hand scale) for $\alpha_{b}=\pi / 2$. We conclude that the cross section for $\alpha_{b}=\pi / 2$ is unobservably small, and so there is no chance of extracting the spacetime structure of the bottom Higgs Yukawa interaction or for that matter even testing for consistency with its SM expectation. Although this is only of academic interest, we see from the right panel that the dashed red histogram for $\alpha_{b}=0$ with the $Z Z h$ coupling switched off essentially tracks the green, dot-dashed histogram for $\alpha_{b}=\pi / 2$. This is again exactly in keeping with what we would expect from the chirality arguments of Sec. II. We have verified this for several other kinematic distributions, but do not show it for brevity. We again conclude that
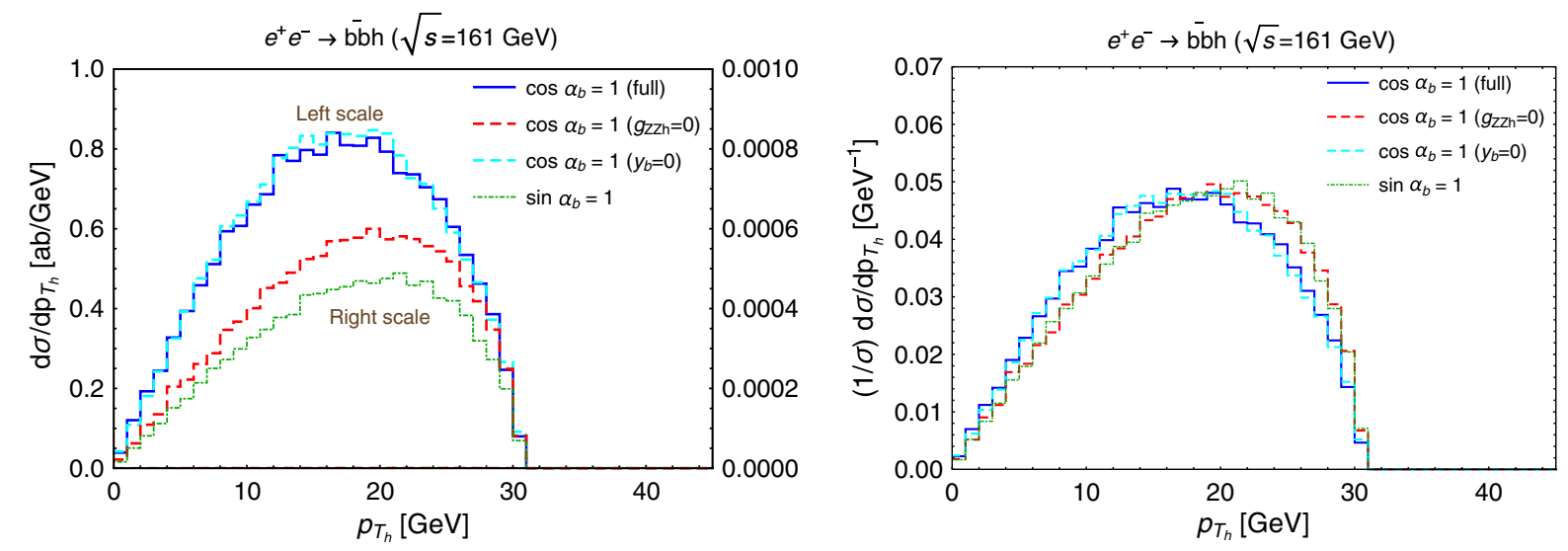

FIG. 3. The left panel shows the differential distribution of $p_{T_{h}}$ produced via $e^{+} e^{-} \rightarrow b \bar{b} h$ at an electron-positron collider with $\sqrt{s}=161 \mathrm{GeV}$ for $\alpha_{b}=0$ and $\alpha_{b}=\pi / 2$. As in Fig. 2, we show three histograms for $\alpha_{b}=0$ and one for $\alpha_{b}=\pi / 2$. The dot-dashed green histogram for $\alpha_{b}=\pi / 2$ and the dashed red histogram for $\alpha_{b}=0$ are to be read on the right-hand scale. The same distributions, but normalized to unity, are shown in the right panel. The total cross sections for $\cos \alpha_{b}=1$ and $\sin \alpha_{b}=1$ cases are 17.2 and $9.75 \times 10^{-3} \mathrm{ab}$, respectively. 
the difference between the $\alpha_{b}=0$ and $\pi / 2$ cases arises (for example the green and the red lines in the left panel) only from the overall normalization which is of no practical use since the coupling of the pseudoscalar particle is not known a priori.

For both the LHC and an $e^{+} e^{-}$collider, we saw that any discernible differences between the scalar and pseudoscalar cases arose only from the additional ZZh coupling. The reader may well wonder whether this occurred because the large gauge coupling of the SM Higgs boson masked the tiny bottom Yukawa coupling. To assure ourselves that this is not the essential reason, we turn to the examination of whether it is possible to determine the spacetime structure of the bottom-quark Yukawa couplings of a hypothetical spin-zero particle $X$ with sizeable couplings to the $b$ quark but which does not develop a vacuum expectation value and has no mixing with the Higgs boson, so that the $Z Z X$ coupling is absent.

\section{B. Yukawa interactions of a hypothetical scalar}

We now turn to the prospects for determining the spacetime structure of the Yukawa interactions of a hypothetical spin-zero particle $X$ with sizeable couplings to bottom quarks. Since our main purpose here is again to examine the impact of the chirality protection mechanism, for definiteness we fix $m_{X}=100 \mathrm{GeV}$, and allow $y_{b b X}$ to be as large as possible. The most generic constraint on the Yukawa coupling of $X$ comes from the nonobservation of an excess of events in a CMS search for $X \rightarrow b \bar{b}$ events accompanied by at least one additional $b$ jet at the $8 \mathrm{TeV}$ LHC [27]. We note that there are LHC13 analyses constraining the couplings of $X$, but these do not extend down to $m_{X}=100 \mathrm{GeV}$. For definiteness, we fix the Yukawa coupling $y_{b b X}=0.7$, compatible with the CMS LHC8 constraints for $m_{X}=100 \mathrm{GeV}$ [28]. ${ }^{3}$

We focus our attention on $e^{+} e^{-}$colliders where precision measurements offer the best hope for distinguishing between a "scalar" $\left(\alpha_{b}=0\right)$ and a "pseudoscalar" $\left(\alpha_{b}=\pi / 2\right) X$ boson. We assume that the $X Z Z$ coupling vanishes because $X$ does not develop a vacuum expectation value, and also does not mix with the SM Higgs boson. We consider $e^{+} e^{-} \rightarrow b \bar{b} X$ at $\sqrt{s}=161 \mathrm{GeV}$, where we expect essentially no physics background from SM $2 \rightarrow 2$ processes, but potentially important backgrounds from $Z b \bar{b}$ and $h b \bar{b}$ production, depending on the $m_{b b}$ mass resolution that is attainable. The CERN FCC and the CEPC being envisioned for construction expect to accumulate 3.8 and $1.3 \mathrm{ab}^{-1} / \mathrm{yr}$ of integrated luminosity, respectively, at each of

\footnotetext{
${ }^{3}$ We mention that hypothetical spin-zero mediators that couple dark matter to SM fermions have been a subject of many studies. In such scenarios, the $y_{b b X}$ coupling may be much more severely constrained using LHC data since invisible $X$ decays can lead to $\mathbb{E}_{T}$ events [29]. Such model-specific considerations are, however, irrelevant for our purposes.
}

two intersection regions. Before any efficiency and acceptance considerations, we would expect several hundred $e^{+} e^{-} \rightarrow b \bar{b} X$ per year at these facilities for $y_{b b X}=0.7$.

We show parton-level results for distributions of various kinematic observables from $e^{+} e^{-} \rightarrow b \bar{b} X$ production, assuming unpolarized electron and positron beams, in Fig. 4. The solid, blue histogram is for $\alpha_{b}=0$ while the dashed, red histogram is for $\alpha_{b}=\pi / 2$. We show results only for the shapes of the distributions because the coupling $y_{b b x}$ which fixes the normalization is clearly not known a priori. Here, $\theta_{b b}$ is the polar angle of the $b \bar{b}$ system in the $e^{+} e^{-}$center-of-mass frame with respect to the direction of the electron beam, while $\phi^{*}$ is the azimuthal angle of $b$ in the $b \bar{b}$ rest frame. In other words, $\phi^{*}$ is the angle between the plane formed by $e^{+}, e^{-}$and $X$ and the plane formed by the $b, \bar{b}$ and $X$ measured in the $b \bar{b}$ rest frame. We recognize that, in practice, it will be difficult to know the $b$ and $\bar{b}$ directions (even if we can use kinematics to distinguish between the primary bottom quarks from the secondary bottoms produced by decay of the $X$ ). Our point, however, is that even if we ignore these practical difficulties, we see from the figure that the distributions are virtually identical (within expected statistics, even ignoring any background) for $\alpha_{b}=0$ and $\alpha_{b}=\pi / 2$. This is, of course, completely in keeping with what we would expect from the chirality considerations of Sec. II.

\section{THRESHOLD BEHAVIOR OF $b \bar{b} X$ PRODUCTION}

The illustrative examples of the previous section serve to confirm that the chirality protection mechanism of Sec. II precludes the possibility of determining $\alpha_{b}$ in high energy processes where the typical process subenergy is much larger than $m_{b}$, for both the SM Higgs boson or for a hypothetical scalar with large couplings to the bottom quark. This naturally suggests that in order to obtain sensitivity to $\alpha_{b}$, the kinematics needs to be such that $b$-quark mass effects are not negligible; i.e., we are close to the reaction threshold, where the reaction products are essentially nonrelativistic.

The threshold dependence of cross sections for $2 \rightarrow 2$ reactions, it is well known, can be extracted using partial wave analysis because, in the center-of-mass system, the associated three-momentum is small so that the contribution from the lowest partial wave dominates. A similar analysis can be performed for the three-body final state, which is characterized by two relative orbital angular momenta. We have the relative orbital momentum $\ell_{b \bar{b}}$ in the rest frame of the $b \bar{b}$ system (which we denote by $B$ ), and also the relative orbital angular momentum $L$ of the $B X$ system in its rest frame which, of course, is also the centerof-mass frame of the three-body final state. Assuming that the final state particles are free particles and well approximated by undistorted plane waves, Moskal et al. [30] have 

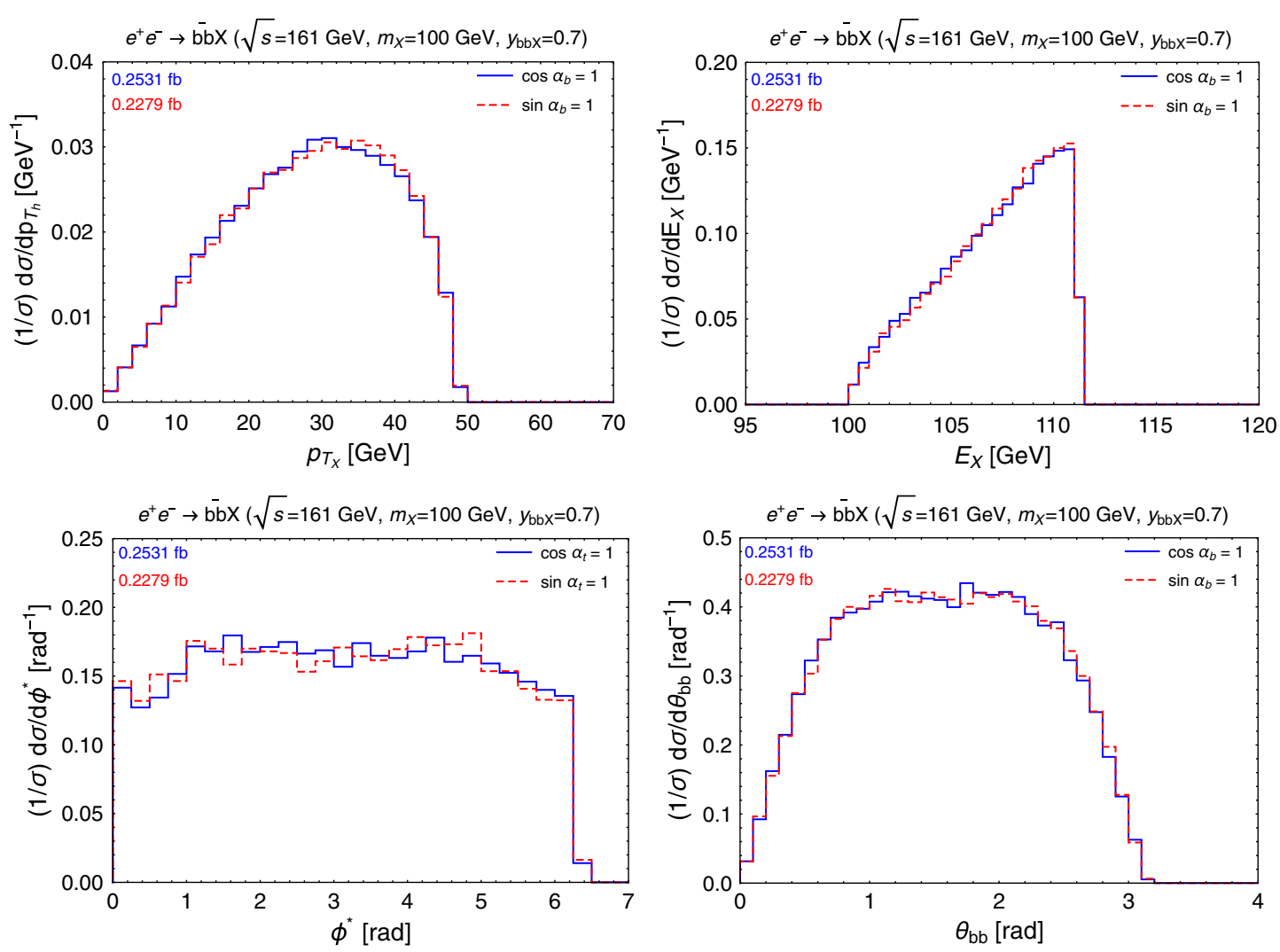

FIG. 4. Differential distributions for kinematic variables, $p_{T_{X}}$ (upper left), $E_{X}$ (upper right), $\phi^{*}$ (lower left), $\theta_{b \bar{b}}$ (lower right) for the process $e^{+} e^{-} \rightarrow b \bar{b} X$ for (unpolarized initial beams) at a center-of-mass energy $\sqrt{s}=161 \mathrm{GeV}$, where $X$ is a spin-zero particle. All distributions are normalized to unity. We take $m_{X}=100 \mathrm{GeV}$ and the Yukawa coupling to bottom quarks $y_{b b X}=0.7$.

shown that the threshold energy dependence of the cross section in the $\left(L, \ell_{b \bar{b}}\right)$ partial wave is given by

$$
\sigma_{L \ell_{b \bar{b}}} \propto q_{\max }^{2 L+2 \ell_{b \bar{b}}+4}
$$

where $q_{\max }$, the maximum momentum of the $X$ is given by

$$
q_{\max }^{2}=\lambda\left(s, 4 m_{b}^{2}, m_{X}^{2}\right) / 4 s .
$$

Here, $\sqrt{s}$ is the center-of-mass energy, and $\lambda(x, y, z) \equiv x^{2}+$ $y^{2}+z^{2}-2 x y-2 y z-2 x z$. It is straightforward to show that close to the reaction threshold, ${ }^{4}$

$$
\sigma_{L \ell_{b \bar{b}}} \propto\left(1-\frac{m_{X}}{\sqrt{s}}-2 \frac{m_{b}}{\sqrt{s}}\right)^{L+\ell_{b \bar{b}}+2} .
$$

We stress that in obtaining this we have used nonrelativistic expressions only for the final state wave functions. That the initial state particles, be they electrons and positrons or even photons, are relativistic, is irrelevant for our analysis. We can use (3) to obtain the threshold behavior of the total

\footnotetext{
${ }^{4}$ Of course, the $b$ quarks are not produced as free particles and hadronize into bottom mesons. The dependence shown in Eq. (3) holds as long as we are not so close to the threshold that the meson binding energy is relevant and not yet so far that the nonrelativistic approximation used to derive the threshold behavior becomes invalid.
}

cross section from various initial states since this is dominated by the lowest values of the exponent $L+\ell_{b \bar{b}}+$ 2 that is allowed by symmetries and any other dynamical considerations (see below).

Since the space parity of a Dirac particle-antiparticle fermion pair in the relative orbital angular momentum state $\ell_{f \bar{f}}$ is given by $(-1)^{\ell_{f \bar{f}}+1}$, and the corresponding charge conjugation parity in the total spin state $S_{f \bar{f}}$ is given by

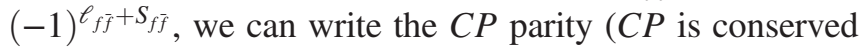
by all relevant interactions) of the $X b \bar{b}$ system [where, abusing notation, we denote $X$ by $h(A)$ for $\alpha_{b}=0(\pi / 2)$ ] as

$$
C P_{h b \bar{b}}=(-1)^{L+S_{b \bar{b}}+1}, \quad C P_{A b \bar{b}}=(-1)^{L+S_{b \bar{b}}} .
$$

In order to proceed further, we note that in the process $e^{+} e^{-} \rightarrow X b \bar{b}$, the final state occurs via an intermediate (virtual) $Z$, and so has total angular momentum $J_{X b \bar{b}}=1$ and $C P_{X b \bar{b}}=1 . C P$ conservation then implies that

$$
\begin{array}{lll}
(-1)^{L}(-1)^{S_{b \bar{b}}}=-1 & \text { for } X=h, & \text { and } \\
(-1)^{L}(-1)^{S_{b \bar{b}}}=+1 & \text { for } X=A . &
\end{array}
$$

For $L=0$, this then implies that

$$
S_{b \bar{b}}=1 \quad \text { for } X=h, \quad S_{b \bar{b}}=0 \quad \text { for } X=A \text {. }
$$



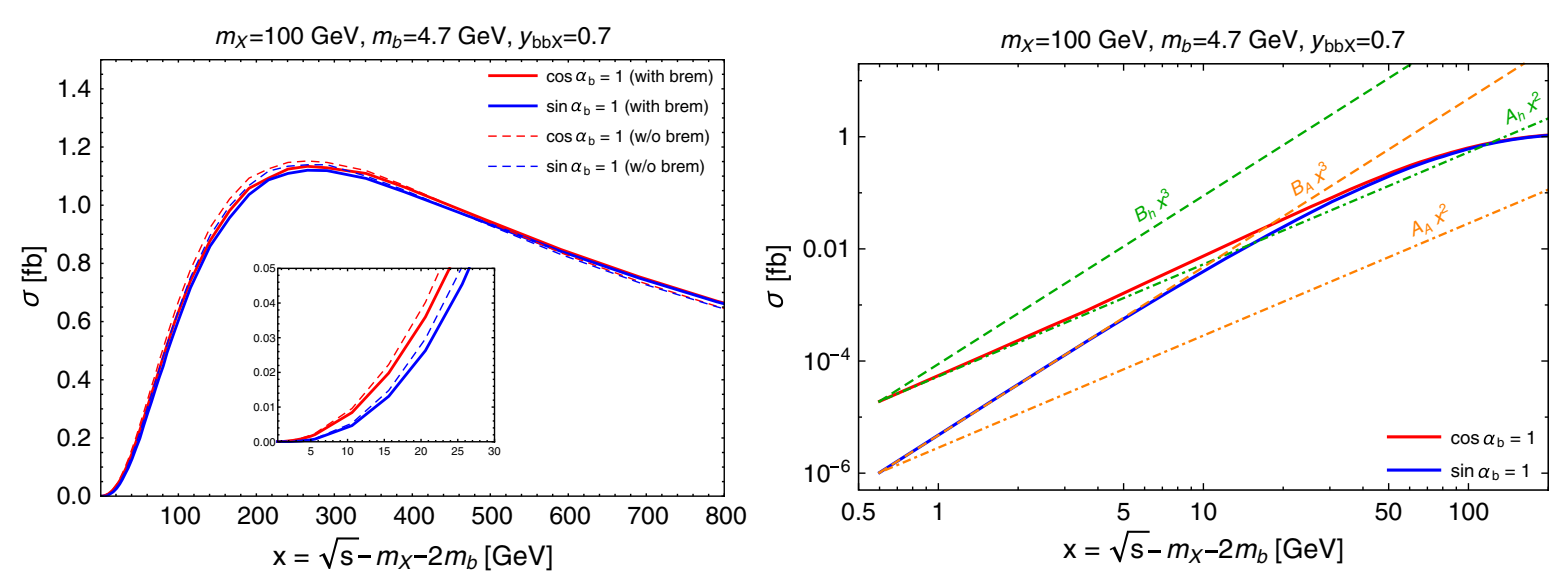

FIG. 5. Cross section for $e^{+} e^{-} \rightarrow b \bar{b} X$ process (with unpolarized beams) for $\cos \alpha_{b}=1$ and $\sin \alpha_{b}=1$, taking $m_{X}=100 \mathrm{GeV}$ and $y_{b b X}=0.7$ versus $x=\sqrt{s}-m_{X}-2 m_{b}$, including beamstrahlung effects on a linear scale (left frame). We also show the same cross section using a log scale (right frame) to show the behavior close to the threshold, $x=0$, along with straight lines that illustrate quadratic and cubic rise of the cross section. The inset on the left frame shows the same cross section with (solid) and without (dashed) beamstrahlung in linear scale.

If, in addition, $\ell_{b \bar{b}}=0$, we conclude that the total angular momentum of the $b \bar{b}$ system is given by

$$
\begin{array}{ll}
J_{b \bar{b}}=1 & \text { for } X=h, \\
J_{b \bar{b}}=0 & \text { for } X=A .
\end{array}
$$

However, $J_{b \bar{b}}=0$ and $L=0$, together are incompatible with angular momentum conservation given that the dynamics requires that the $b \bar{b} A$ final state comes from a spin- $1 Z^{*}$. This is not an issue for $b \bar{b} h$ production, as can be seen from Eq. (4). We thus conclude from Eq. (3) that close to the production threshold,

$$
\sigma\left(e^{+} e^{-} \rightarrow b \bar{b} h\right) \sim\left(\sqrt{s}-2 m_{b}-m_{h}\right)^{2},
$$

while

$$
\sigma\left(e^{+} e^{-} \rightarrow b \bar{b} A\right) \sim\left(\sqrt{s}-2 m_{b}-m_{A}\right)^{3} .
$$

We stress that $C P$ and angular momentum conservation are, by themselves, not sufficient to yield the threshold behavior of the cross sections in Eqs. (6) and (7). It is crucial to use the fact that the final state arises from a virtual $Z^{*}$, and so has $J_{X b \bar{b}}=1$ and $C P=+1$. This is what we had referred to as "other dynamical considerations" at the end of the paragraph containing Eq. (3).

At first glance, the different powers in Eqs. (6) and (7) appear to be at odds with the chirality protection mechanism of Sec. II. We have, however, extracted the threshold behavior of these cross sections from the lengthy expressions for the full calculation of $e^{+} e^{-} \rightarrow t \bar{t}+h / A$ production [31] and find that, at the threshold for the $b \bar{b}+h / A$ case,

$$
\begin{aligned}
& \sigma\left(e^{+} e^{-} \rightarrow b \bar{b} h\right) \sim\left(\sqrt{s}-m_{h}\right)\left(\sqrt{s}-2 m_{b}-m_{h}\right)^{2}, \quad \text { and } \\
& \sigma\left(e^{+} e^{-} \rightarrow b \bar{b} A\right) \sim\left(\sqrt{s}-2 m_{b}-m_{A}\right)^{3} .
\end{aligned}
$$

We see that the explicit calculation is consistent with the expected threshold behavior in Eqs. (6) and (7). Moreover, we see that, in the chiral limit $m_{b} \rightarrow 0$, the prefactor $(\sqrt{s}-$ $\left.m_{h}\right)$ present in the expression for $\sigma\left(e^{+} e^{-} \rightarrow b \bar{b} h\right)$ above combines with the second factor to yield the same behavior for the $b \bar{b} h$ and $b \bar{b} A$ production, in agreement with what we would expect from the chirality protection mechanism.

To illustrate the threshold behavior, we show the centerof-mass energy dependence of the cross section for $e^{+} e^{-} \rightarrow b \bar{b} X$ production for $\alpha_{b}=0$ (solid red) and $\alpha_{b}=$ $\pi / 2$ (solid blue) in the left frame of Fig. 5, plotting it versus the kinetic energy release $x=\sqrt{s}-m_{X}-2 m_{b}$. As before, we fix $m_{X}=100 \mathrm{GeV}, y_{b b X}=0.7$ and assume that there is no $Z Z X$ coupling. We include beamstrahlung effects using the Kuraev-Fadin distribution function [32] for an electron inside the electron as described in Sec. 2 of Ref. [33]. Beamstrahlung makes only a small shift of the normalization without any appreciable change in the threshold behavior from our expectation as we will see shortly. We do not include any beamstrahlung effects as these will depend on the as yet undetermined details of the beam configurations, but we expect this would not have a qualitative effect on the following discussion. We see from the figure that the cross section is essentially independent of $\alpha_{b}$, just as we would expect from the discussion of Sec. II. ${ }^{5}$

\footnotetext{
${ }^{5}$ We emphasize that only the energy dependence can be used to distinguish between the two cases because the coupling $y_{b b X}$ is completely unknown. Although this is completely academic, we note that for the integrated luminosities we may anticipate at CEPC or FCC with $\sqrt{s} \sim 240-250 \mathrm{GeV}$, even the normalization difference seen in the figure would be difficult to distinguish, since we may expect no more than a few thousand signal events per year per interaction point before any acceptance cuts and detector efficiencies are included.
} 
Turning to the right-hand frame, we see that sufficiently close to the threshold, the cross section indeed exhibits the quadratic [cubic] variation that we anticipated in Eq. (6) [Eq. (7)] for $X=h(X=A)$. Of course, this makes sense only for $x$ larger than a few $\mathrm{GeV}$ so that we can neglect bottom meson binding effects. Such a study will also require scanning the cross section close to the reaction threshold. For $b \bar{b} A$ production, the cubic dependence on $x$ persists for $\lesssim 20 \mathrm{GeV}$ beyond the threshold, where the cross section is $\sim 5-20$ ab. In contrast, we see that the cross section for $b \bar{b} h$ production begins to deviate from the expected quadratic dependence on $x$ even $\sim 5 \mathrm{GeV}$ above threshold, where the cross section is below 2 ab. It thus appears that with integrated luminosities of about $10 \mathrm{ab}^{-1}$ (i.e., $\sim 1 \mathrm{ab}^{-1}$ per point) may allow confirmation of the expected threshold behavior of $b \bar{b} A$ production, but significantly higher integrated luminosities will be needed for the corresponding study of $b \bar{b} h$ production. We caution that these projections should be regarded only as a qualitative indication of what might be possible at $e^{+} e^{-}$ future colliders since we have assumed a particular value of $y_{b b X}$, and not included geometric acceptances or experimental efficiencies, or examined analysis cuts that may be needed to separate the signal from background. ${ }^{6}$ Our point here is only that such a threshold analysis may be worthy of further assessment should a new spin-zero particle that couples to bottom quarks be discovered. A scan over the $b \bar{b} X$ production threshold may well be the only way to reveal the spacetime structure of the bottom Yukawa coupling of the new spin-zero particle.

Before closing this section, we note that we can use similar reasoning to extract the threshold behavior of $b \bar{b} X$ production from other initial states. For instance, for $\gamma \gamma \rightarrow b \bar{b} X$, both $C$ and $C P$ are separately conserved, but the final state is not dynamically constrained to have $C P=1$. We find that $L=\ell_{b \bar{b}}=0$ is consistent with $C$ conservation if $S_{b \bar{b}}=0$ since $C_{\gamma \gamma}=+1$. $C P$ conservation then implies that $b \bar{b} h(b \bar{b} A)$ production is allowed as long as the initial photons are in the $C P$ odd (even), $J=0$ state. We conclude that

$\sigma(\gamma \gamma \rightarrow b \bar{b} X) \sim\left(\sqrt{s}-2 m_{b}-m_{X}\right)^{2}, \quad X=h, A$.

We have verified that the explicit computation yields this threshold behavior of the cross sections.

The threshold behavior for $b \bar{b} X$ production from $q \bar{q}$ initial states is, of course, identical to that from the electron-positron initial state, with the virtual gluon

\footnotetext{
${ }^{6} \mathrm{We}$ also repeat that we have assumed that there is no $X Z Z$ coupling, reminding the reader that in the SM the amplitude containing the $h Z Z$ coupling overwhelms $b \bar{b} h$ production.
}

playing the role of the $Z^{*}$, and constraining the final state to be $C P$ even. Production from the $g g$ initial state is more complicated than from the $\gamma \gamma$ initial state because now additional amplitudes are present because of the existence of the three-gluon vertex. In any case all this is only of academic interest, since at a hadron collider it will almost certainly be impossible to experimentally study the threshold behavior of the production cross section, let alone disentangle the various subprocesses from each other.

\section{TOP PAIR PRODUCTION IN ASSOCIATION WITH A SPIN-ZERO PARTICLE}

We have seen in Sec. III A that the shape of the $p_{T h}$ distribution can be used to distinguish between $\alpha_{t}=0$ and $\alpha_{t}=\pi / 2$ at the LHC [14], while the chirality protection mechanism precludes the possibility for analogously pinning down $\alpha_{b}$. We could equally well have used the $M_{\bar{t} \bar{t} h}$ distribution to distinguish between $\alpha_{t}=0$ and $\alpha_{t}=\pi / 2$ as can be seen from the top panel of Fig. 6. What may be somewhat of a surprise is that the SM Higgs mass is accidentally close to a sweet spot for distinguishing between the two values of $\alpha_{t}$. This may be seen in the bottom frames of Fig. 6, where we show the $M_{t t h}$ distributions for the same two values of $\alpha_{t}$, but for $m_{h}=$ 250 and $400 \mathrm{GeV}$. It is clear that for the larger values of $m_{h}$ distinction between $\alpha_{t}=0$ and $\alpha_{t}=\pi / 2$ becomes much more challenging. ${ }^{7}$ Although this is somewhat unrelated to the main subject of the paper, we remark that it would be erroneous to infer that one can use kinematic distributions to extract the spacetime structure of $t \bar{t} X$ couplings, regardless of the mass of $X$. For $m_{X} \gg m_{t}$, the distinction between even the extreme cases, $\alpha_{t}=0$ and $\alpha_{t}=\pi / 2$ vanishes, in accord with the chirality protection argument.

Prior to closing this discussion, we mention that we have also checked that the variation of the $e^{+} e^{-} \rightarrow t \bar{t} h$ and $\gamma \gamma \rightarrow$ $t \bar{t} h$ cross sections with the center-of-mass energy $\sqrt{s}=$ $M_{t t h}$ also exhibit the same qualitative feature: The energy dependence of the cross section becomes insensitive to the spacetime structure of the top-quark Yukawa coupling in Eq. (1) for large values of $m_{h}$. This may be understood by noting that the $t \bar{t} h$ production cross section (or in the case of the LHC, the hard scattering cross section as a function of parton center-of mass energy $\hat{s}$ instead of $s$ ) takes the form,

$\sigma\left(s, m_{t}, m_{h}\right)=\frac{1}{s}\left(1-\frac{2 m_{t}}{\sqrt{s}}-\frac{m_{h}}{\sqrt{s}}\right)^{p} \times G\left(\frac{m_{t}}{\sqrt{s}}, \frac{m_{h}}{\sqrt{s}}\right)$,

where we have separated the factor to make explicit the threshold energy dependence discussed in Sec. IV. Any

\footnotetext{
${ }^{7}$ We have checked that this is also the case for the $p_{T h}$ distribution in $p p \rightarrow t \bar{t} h$ production at the LHC.
} 

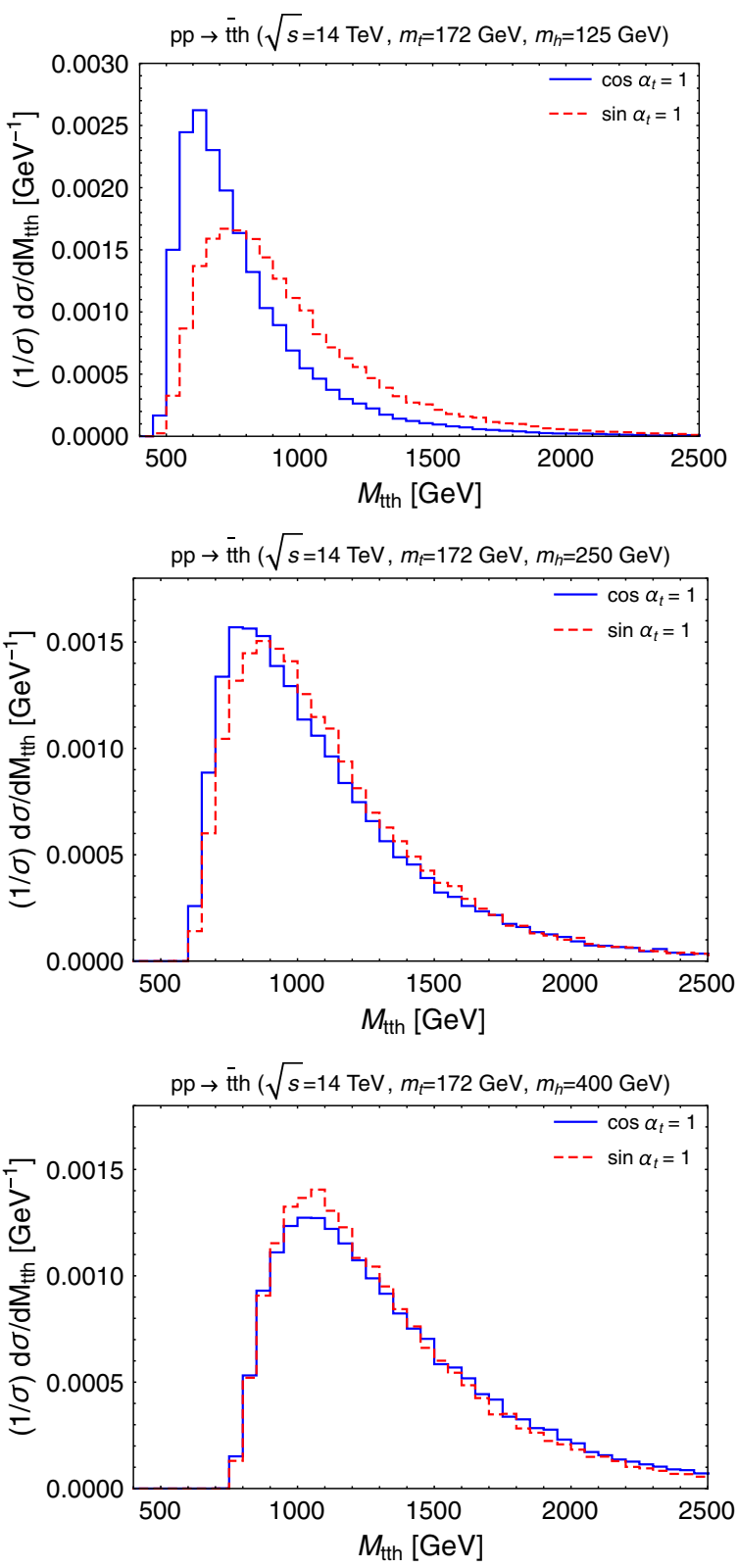

FIG. 6. In the top panel we show the invariant mass distributions for $t \bar{t} h$ production at the LHC, for $m_{h}=125 \mathrm{GeV}$. In the two lower panels we show invariant mass distributions for $m_{h}=$ 250 and $400 \mathrm{GeV}$.

dependence of the cross section on $\alpha_{t}$ enters via the dimensionless function $G$, which encodes the information of the underlying dynamics. The chirality protection mechanism implies that the dependence on $\alpha_{t}$ must vanish if $\frac{m_{t}}{\sqrt{s}} \rightarrow 0$. Since we know that the cross section in Eq. (9) does not exhibit singular behavior as $m_{t} \rightarrow 0$, its sensitivity to $\alpha_{t}$ is suppressed by $m_{t} / \sqrt{s}<m_{t} / m_{h}$, accounting for the behavior seen in Fig. 6, and for the fact that this behavior persists for $t \bar{t} h$ production from $\gamma \gamma$ as well as $e^{+} e^{-}$initial states. ${ }^{8}$

\footnotetext{
${ }^{8}$ The argument readily extends to the $p_{T h}$ distribution, as long as this distribution is not singular behavior as $p_{T} \rightarrow 0$.
}

\section{SUMMARY AND CONCLUDING REMARKS}

The original motivation for this study was to examine strategies to determine the spacetime structure of the renormalizable bottom-quark Yukawa interaction with the putative Higgs boson discovered at CERN. Are these scalar, pseudoscalar or somewhere in between? We found that adapting strategies that have been suggested in the literature for the determination in case of the top quark simply does not work for the bottom-quark case.

We traced the underlying reason to the fact that by making suitable chiral transformations, it is possible to continuously transform between scalar and pseudoscalar Yukawa interactions with any spin-zero particle, without affecting its interactions with SM vector bosons. These chiral transformations alter only the quark mass term. If the quark mass vanishes (but the Yukawa coupling is held fixed), it is only a matter of convention whether we call the interaction scalar or pseudoscalar, and no experimental observable is affected by this change of description. This was what we dubbed the chirality protection mechanism in Sec. II. In practice, any differences between scalar versus pseudoscalar interactions are suppressed as long as all relevant subprocess energy scales are large compared to $m_{b}$, so that the bottom quarks are relativistic.

Motivated by the fact that a study of $t \bar{t} h$ production had been shown to allow for promising ways to elucidate the spacetime structure of the top-quark Yukawa coupling, in Sec. III we examined $b \bar{b} h$ production both at the LHC as well as at an electron-positron collider, and evaluated several kinematic distributions to quantify the efficacy of the chirality protection mechanism. We found that, even at the parton level and without any detector resolution effects, the differences are too small to allow distinction between scalar and pseudoscalar Yukawa interactions: More realistic simulations would reduce these even further.

There are several factors that make the study of the SM bottom-quark Yukawa interaction more difficult compared to that of the top quark. First, the bottom Yukawa coupling is small, so one suffers from low rates. Second, because the Yukawa coupling is small, the contribution where the $h$ is radiated from the (virtual) $Z$ dominates $e^{+} e^{-} \rightarrow b \bar{b} h$ production and overwhelms the contributions of interest that involve the bottom-quark Yukawa coupling. ${ }^{9}$ We found that in high energy processes where the bottom quarks are relativistic, the chirality protection mechanism precludes the possibility of determining the spacetime structure of the renormalizable interactions, even for a hypothetical spinzero particle with a large coupling to bottom quarks; see Sec. III B. As discussed in Sec. IV, close to the reaction threshold for $e^{+} e^{-} \rightarrow b \bar{b} X$ production where the

\footnotetext{
${ }^{9}$ In addition, the decay products of the top quark retain information on the polarization of the parent top, and so provide an additional handle. Bottom quark decays mostly do not preserve polarization information.
} 
bottom-quark mass effects are significant, the chirality protection mechanism ceases to be effective, leading to a potentially observable difference in the threshold behavior for $X=h$ and $X=A$ for integrated luminosities of a few $\mathrm{ab}^{-1}$ per year, and a Yukawa coupling significantly larger than in the SM.

Are there other ways of differentiating between scalar versus pseudoscalar couplings of $b$ quarks to spin-zero particles? As we showed, any differences vanish in the limit of vanishing $b$-quark mass. One possibility might be the precise determination of the rate for $X \rightarrow b \bar{b}$ decay which is suppressed by $\beta^{3}$ for $X=h$ but by $\beta$ for $X=A$, where $\beta=\left(1-\frac{4 m_{b}^{2}}{m_{X}^{2}}\right)^{1 / 2}$. We make no representation as to whether this might be feasible, but mention it more to highlight the difficulty of determining the spacetime structure of the coupling.

In summary, our examination of the prospects for determining the spacetime structure of the Higgs boson Yukawa interaction of bottom quarks in the SM led us to largely negative conclusions. While the smallness of the SM Yukawa coupling is an important factor, the real underlying reason for this is that chirality considerations imply that any difference between scalar and pseudoscalar interactions vanishes in the limit $m_{b} \rightarrow 0$, and so is unobservably small in high energy processes where subprocess energy scales are set by the mass of the spin-zero particle, typically much larger than $m_{b}$. Indeed we found that the difference between scalar and pseudoscalar interactions remains unobservable even for a hypothetical spinzero particle with large Yukawa couplings to bottom quarks. The energy dependence of the reaction $e^{+} e^{-} \rightarrow$ $b \bar{b} X$ production close to the reaction threshold, where the bottom quarks are nonrelativistic offers the best hope for distinguishing between scalar and pseudoscalar couplings of $X$ to bottom quarks.

\section{ACKNOWLEDGMENTS}

This work was supported in part by the U.S. Department of Energy, Office of High Energy Physics Grant No. desc0010504. X. T. thanks the Centre for High Energy Physics, Indian Institute of Science Bangalore, where this project was begun for their hospitality, and also the Infosys Foundation for financial support that made his visit to Bangalore possible. The work of R. G. is supported by the Department of Science and Technology, India under Grant No. SR/S2/JCB-64/2007.
[1] G. Aad et al. (ATLAS Collaboration), Phys. Lett. B 716, 1 (2012); S. Chatrchyan et al. (CMS Collaboration), Phys. Lett. B 716, 30 (2012).

[2] V. Khachatryan et al. (CMS Collaboration), Phys. Rev. D 92, 012004 (2015); G. Aad et al. (ATLAS Collaboration), Eur. Phys. J. C 75, 476 (2015); 76, 152(E) (2016); V. Khachatryan et al. (CMS Collaboration), Phys. Lett. B 759, 672 (2016); G. Aad et al. (ATLAS Collaboration), Eur. Phys. J. C 76, 658 (2016); A. M. Sirunyan et al. (CMS Collaboration), Phys. Lett. B 775, 1 (2017); M. Aaboud et al. (ATLAS Collaboration), J. High Energy Phys. 03 (2018) 095; A. M. Sirunyan et al. (CMS Collaboration), Phys. Rev. D 99, 112003 (2019); A. M. Sirunyan et al. (CMS Collaboration), arXiv:1903.06973.

[3] G. Aad et al. (ATLAS Collaboration), J. High Energy Phys. 04 (2015) 117; A. M. Sirunyan et al. (CMS Collaboration), Phys. Lett. B 779, 283 (2018).

[4] M. Aaboud et al. (ATLAS Collaboration), Phys. Lett. B 786, 59 (2018); A. M. Sirunyan et al. (CMS Collaboration), Phys. Rev. Lett. 121, 121801 (2018).

[5] M. Aaboud et al. (ATLAS Collaboration), Phys. Lett. B 784, 173 (2018); A. M. Sirunyan et al. (CMS Collaboration), Phys. Rev. Lett. 120, 231801 (2018).

[6] A. M. Sirunyan et al. (CMS Collaboration), Phys. Rev. Lett. 122, 021801 (2019).

[7] F. Boudjema, R. M. Godbole, D. Guadagnoli, and K. A. Mohan, Phys. Rev. D 92, 015019 (2015); G. Brooijmans et al., arXiv:1405.1617.
[8] J. F. Gunion, B. Grzadkowski, and X. G. He, Phys. Rev. Lett. 77, 5172 (1996).

[9] P. S. Bhupal Dev, A. Djouadi, R. M. Godbole, M. M. Muhlleitner, and S. D. Rindani, Phys. Rev. Lett. 100, 051801 (2008); R. M. Godbole, C. Hangst, M. Muhlleitner, S. D. Rindani, and P. Sharma, Eur. Phys. J. C 71, 1681 (2011); K. Desch, A. Imhof, Z. Was, and M. Worek, Phys. Lett. B 579, 157 (2004).

[10] J. R. Dell'Aquila and C. A. Nelson, Nucl. Phys. B320, 61 (1989); S. Berge, W. Bernreuther, and J. Ziethe, Phys. Rev. Lett. 100, 171605 (2008); A. Askew, P. Jaiswal, T. Okui, H. B. Prosper, and N. Sato, Phys. Rev. D 91, 075014 (2015); S. Berge, W. Bernreuther, and S. Kirchner, Phys. Rev. D 92, 096012 (2015); T. Han, S. Mukhopadhyay, B. Mukhopadhyaya, and Y. Wu, J. High Energy Phys. 05 (2017) 128.

[11] J. F. Gunion and X. G. He, Phys. Rev. Lett. 76, 4468 (1996).

[12] A. V. Gritsan, R. Rontsch, M. Schulze, and M. Xiao, Phys. Rev. D 94, 055023 (2016).

[13] J. Ellis, D. S. Hwang, K. Sakurai, and M. Takeuchi, J. High Energy Phys. 04 (2014) 004.

[14] R. Frederix, S. Frixione, V. Hirschi, F. Maltoni, R. Pittau, and P. Torrielli, Phys. Lett. B 701, 427 (2011).

[15] F. Demartin, F. Maltoni, K. Mawatari, B. Page, and M. Zaro, Eur. Phys. J. C 74, 3065 (2014).

[16] S. P. Amor dos Santos et al., Phys. Rev. D 92, 034021 (2015).

[17] S. Amor dos Santos et al., Phys. Rev. D 96, 013004 (2017). 
[18] S. Heinemeyer et al. (LHC Higgs Cross Section Working Group), arXiv:1307.1347.

[19] S. Biswas, R. Frederix, E. Gabrielli, and B. Mele, J. High Energy Phys. 07 (2014) 020.

[20] For a review and more references, see A. Djouadi, Phys. Rep. 457, 1 (2008).

[21] B. Ananthanarayan, S. K. Garg, J. Lahiri, and P. Poulose, Phys. Rev. D 87, 114002 (2013).

[22] K. Hagiwara, K. Ma, and H. Yokoya, J. High Energy Phys. 06 (2016) 048.

[23] A. F. Falk and M. E. Peskin, Phys. Rev. D 49, 3320 (1994).

[24] M. Galanti, A. Giammanco, Y. Grossman, Y. Kats, E. Stamou, and J. Zupan, J. High Energy Phys. 11 (2015) 067.

[25] D. Goncalves, K. Kong, and J. H. Kim, J. High Energy Phys. 06 (2018) 079.

[26] CEPC Study Group, arXiv:1809.00285; D. d'Enterria, arXiv: 1602.05043.
[27] V. Khachatryan et al. (CMS Collaboration), J. High Energy Phys. 11 (2015) 071.

[28] A. M. Sirunyan et al. (CMS Collaboration), J. High Energy Phys. 08 (2018) 113.

[29] M. R. Buckley, D. Feld, and D. Goncalves, Phys. Rev. D 91, 015017 (2015); M. R. Buckley and D. Goncalves, Phys. Rev. Lett. 116, 091801 (2016); M. R. Buckley and D. Goncalves, Phys. Rev. D 93, 034003 (2016); 93, 034003 (2016).

[30] P. Moskal, M. Wolke, A. Khoukaz, and W. Oelert, Prog. Part. Nucl. Phys. 49, 1 (2002).

[31] A. Djouadi, J. Kalinowski, and P. M. Zerwas, Z. Phys. C 54, 255 (1992).

[32] E. A. Kuraev and V. S. Fadin, Yad. Fiz. 41, 733 (1985) [Sov. J. Nucl. Phys. 41, 466 (1985)].

[33] H. Baer, T. Krupovnickas, and X. Tata, J. High Energy Phys. 06 (2004) 061. 